\title{
Mechanistic Targets of Diallyl Trisulfide in Human Breast Cancer Cells Identified by RNA-seq Analysis
}

\author{
Eun-Ryeong Hahm', Su-Hyeong Kim', Sivapar V. Mathan², Rana P. Singh², Shivendra V. Singh ${ }^{1}$ \\ ${ }^{1}$ Department of Pharmacology \& Chemical Biology, and UPMC Hillman Cancer Center, University of Pittsburgh School of \\ Medicine, Pittsburgh, PA, USA, ${ }^{2}$ Cancer Biology Laboratory, School of Life Sciences, Jawaharlal Nehru University, New \\ Delhi, India
}

Diallyl trisulfide (DATS), a metabolic by-product of processed garlic, is highly effective in inhibiting growth of human breast cancer cells in vitro and in vivo, but the underlying mechanisms are still not fully understood. In this study, we performed RNA-seq analyses using luminal-type (MCF-7) and basal-like (MDA-MB-231) human breast cancer cells to identify mechanistic targets of DATS. The Reactome Pathway Analysis revealed upregulation of genes associated with SLIT/ROBO tumor suppressor signaling following DATS treatment in both MCF-7 and MDA-MB-231 cells. However, the expression of SLIT2 and ROBO1 proteins or their downstream target C-X-C motif chemokine receptor 4 was not affected by DATS treatment in both cell lines. The Reactome as well as the Gene Ontology Pathways Analyses of the RNA-seq data from DATS-treated cells indicated downregulation of genes associated with $\mathrm{G}_{2} / \mathrm{M}$ phase cell cycle arrest in comparison with vehicle-treated control cells. Consistent with the RNA-seq data, DATS treatment caused a significant increase in the fraction of the $\mathrm{G}_{2} / \mathrm{M}$ population in both cell lines when compared to corresponding control cells. In addition, Ser10 phosphorylation of histone $\mathrm{H} 3$, a mitotic marker, was also increased significantly following DATS treatment in MCF-7 and MDA-MB-231 cells. These results indicate that while SLIT/ROBO signaling is not affected by DATS treatment, cell cycle arrest likely contributes to the antitumor effect of this phytochemical.

Key Words Breast neoplasms, Allyl compounds, Sulfides, Chemoprevention

\section{INTRODUCTION}

Breast cancer is a leading cause of cancer-related deaths in American females [1]. In addition to the changes in lifestyle, chemoprevention represents a promising approach for reducing the morbidity and mortality associated with breast cancer [2]. Breast cancer is a heterogeneous malignancy broadly grouped into three major subtypes (luminal-type, human epidermal growth factor receptor 2 [Her-2]-enriched, and basal-like), but a clinical chemopreventative intervention is available only for the luminal-type disease [3-5]. Because of their safety, edible plants like Allium (garlic, onion, leek, etc.) and cruciferous vegetables (broccoli, watercress, mustard, etc.) and their bioactive small molecules continue to attract research attention with respect to their chemopreventive potential [6-8].

Epidemiological studies have suggested the beneficial effects of garlic and onion on breast cancer risk [9-11]. In a
French case-control study with 345 patients and controls matched for age and socio-economic status, the breast cancer risk was inversely associated with increased intake of garlic and onions [9]. In another study conducted in Puerto Rico, an inverse association was observed for breast cancer risk with moderate consumption (odds ratio $[\mathrm{OR}]=0.59$ ) and high consumption $(\mathrm{OR}=0.51)$ when compared to low consumption of onion and garlic $\left(P_{\text {trend }}=0.02\right)$ [10]. The study conclusions did not change when stratified by the menopausal status [10]. However, epidemiological evidence of breast cancer risk reduction was not discernible for garlic-supplement in the Netherlands cohort study [12]. A likely variable contributing to this discrepancy could be insufficient levels of bioactive components in the supplement, which was not considered in the study design [12]. Nevertheless, epidemiological evidence is generally supportive of breast cancer risk reduction by dietary intake of garlic and onion [9-11].

The protective effect of garlic and onions on breast cancer

Received May 10, 2021, Revised May 26, 2021, Accepted May 26, 2021

Correspondence to Shivendra V. Singh, E-mail: singhs@upmc.edu, https://orcid.org/0000-0002-3733-144X

Check for updates 
risk is attributable to organosulfur compounds (OSCs), which are generated upon processing (mincing, chewing, etc.) [13]. The primary sulfur compounds in intact Allium vegetables are $\gamma$-glutamyl-S-alk(en)yl-L-cysteines, which are hydrolyzed and oxidized to yield $S$-alk(en)yl-L-cysteine sulfoxide (alliin) [13]. Alliin is odorless and accumulates during storage of the Allium vegetables. Processing of Allium vegetables releases an enzyme (alliinase) that converts alliin to allicin and other alkyl alkane-thiosulfinates [13]. Finally, allicin and related thiosulfinates are decomposed to yield various sulfur compounds including diallyl sulfide (DAS), diallyl disulfide (DADS), diallyl trisulfide (DATS), dithiins, ajoene, etc. [13]. The anticancer potency of the above listed OSCs is in the order DATS > DADS > DAS in cultured breast cancer cells in vitro [14]. Na et al. [15] were the first to demonstrate in vivo growth inhibitory effect of DATS in a therapeutic setting (xenograft model) using luminal-type MCF-7 cells. A very mild regimen of twice weekly oral administration of about $0.9 \mathrm{mg}$ DATS $/ \mathrm{kg}$ body weight for one month exhibited remarkable $(>80 \%)$ inhibition of MCF-7 xenograft growth in athymic mice [15]. Even though we intend to eventually develop DATS itself for chemopre- vention of breast cancer as a point of reference, it is estimated that $1 \mathrm{~g}$ of fresh garlic can yield up to $1.1 \mathrm{mg}$ of DATS [16]. The level of DATS in fresh garlic is relatively higher than that of DAS (0.1 mg) or DADS (up to $0.6 \mathrm{mg}$ ) [16].

Despite promising in vitro and in vivo data on anticancer effects of DATS in breast cancer, the underlying mechanisms are not fully understood. In the study, we compared RNA-seq data using control (vehicle-treated) and DATS-treated MCF7 and MDA-MB-231 human breast cancer cell lines to gain insights into the mechanism(s) by which DATS inhibits the growth of breast cancer cells.

\section{MATERIALS AND METHODS}

\section{Reagents}

DATS (99.2\%) was purchased from LKT Laboratories (St. Paul, MN, USA), dissolved in dimethyl sulfoxide (DMSO; 28 $\mathrm{mmol} / \mathrm{L}$ stock), and stored at $-80^{\circ} \mathrm{C}$ prior to use. Cell culture media were from MediaTech (Manassas, VA, USA). Fetal bovine serum was obtained from Atlanta Biologicals (Norcross, GA, USA). Antibodies against ROBO1 and SLIT2 were from
A

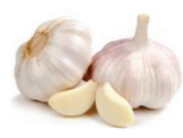<smiles>C=CCSSSCC=C</smiles>

B MCF-7

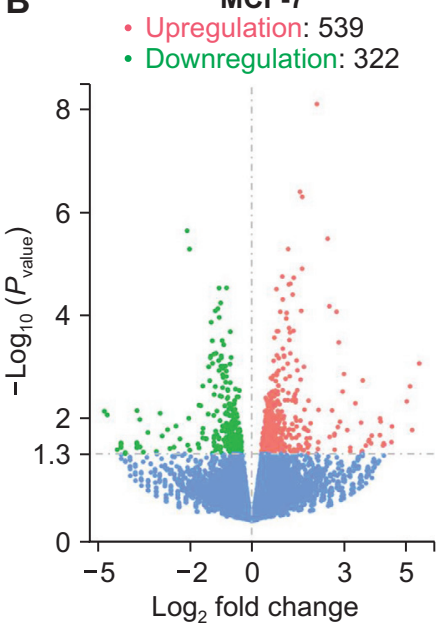

C

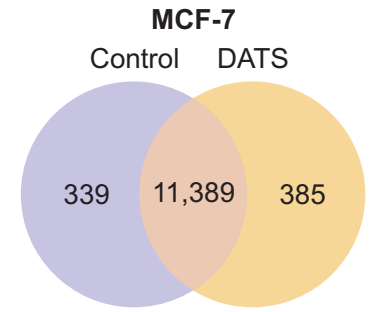

MDA-MB-231

- Upregulation: 644

- Downregulation: 508

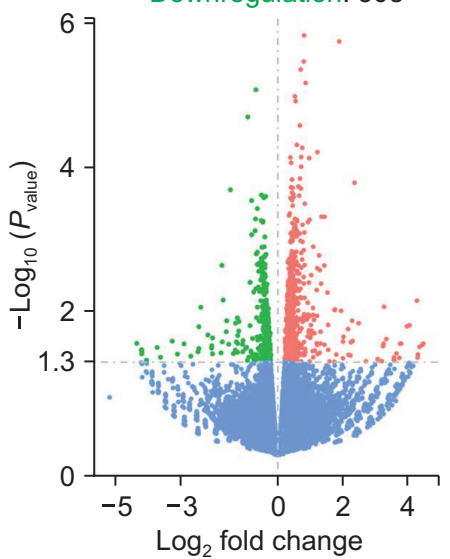

MDA-MB-231

Control DATS

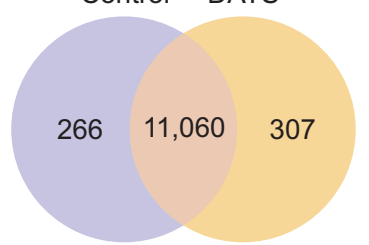

D

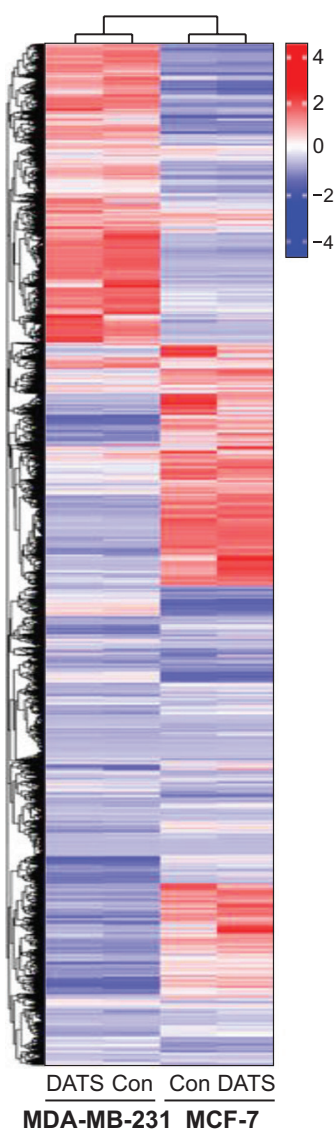

Figure 1. DATS treatment altered breast cancer transcriptomes. (A) Chemical structure of DATS. (B) Volcano scatter plots showing genes upregulated or downregulated in MCF-7 and MDA-MB-231 cells in response to DATS. (C) Venn diagram showing unique and common genes by DATS treatment in MCF-7 and MDA-MB-231 cells. (D) Heatmap diagram visualizing the differentially expressed genes by DATS treatment in MCF-7 and MDA-MB-231 cells. DATS, diallyl trisulfide; MCF-7, michigan cancer foundation-7; MDA-MB-231, MD anderson-metastatic breast-231. 
Proteintech Group (Rosemont, IL, USA), while anti-C-X-C motif chemokine receptor 4 antibody was from Abcam (Cambridge, MA, USA). Alexa Fluor 488-conjugated phospho-(Ser10) histone $\mathrm{H} 3$ antibody was from Cell Signaling Technology (Danvers, MA, USA). Anti-phospho-(Ser10)-histone H3 antibody was from Millipore-Sigma (Burlington, MA, USA). Anti- $\beta$-actin antibody and propidium iodide $(\mathrm{PI})$ were from Sigma (St. Louis, MO, USA).

\section{Cell culture}

MCF-7 and MDA-MB-231 human breast cancer cell lines were purchased from the American Type Culture Collection (Manassas, VA, USA), and each cell line was maintained as suggested by the supplier. These cell lines were last authenti- cated by us in March of 2017.

\section{RNA-seq analysis}

Samples were prepared three times independently. Briefly, MCF-7 and MDA-MB-231 cells were plated in 10-cm dishes at a density of $1 \times 10^{6}$ cells/dish and incubated overnight for attachment. The cells were treated with DMSO (final concentration: $0.07 \%$ ) or $20 \mu \mathrm{mol} / \mathrm{L}$ of DATS for 16 hours. Total RNA was isolated using RNeasy Mini Kit from Qiagen (Germantown, MD, USA). RNA quality determination and RNAseq analysis were performed by Novogene (Sacramento, CA, USA). Other details of RNA-seq analysis were similar as described by us previously [17]. The RNA-seq data were analyzed using the Reactome, Gene Ontology (GO), and
A
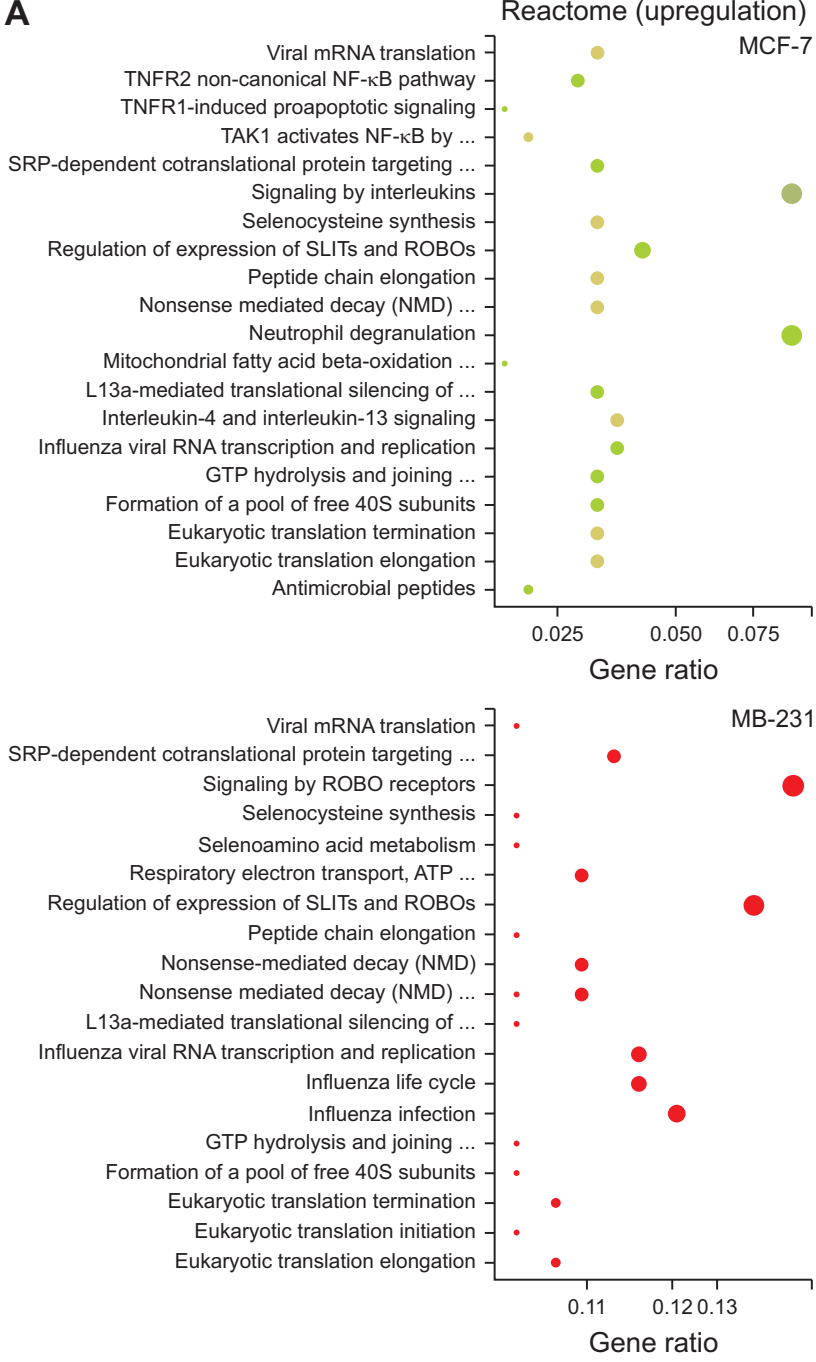

B
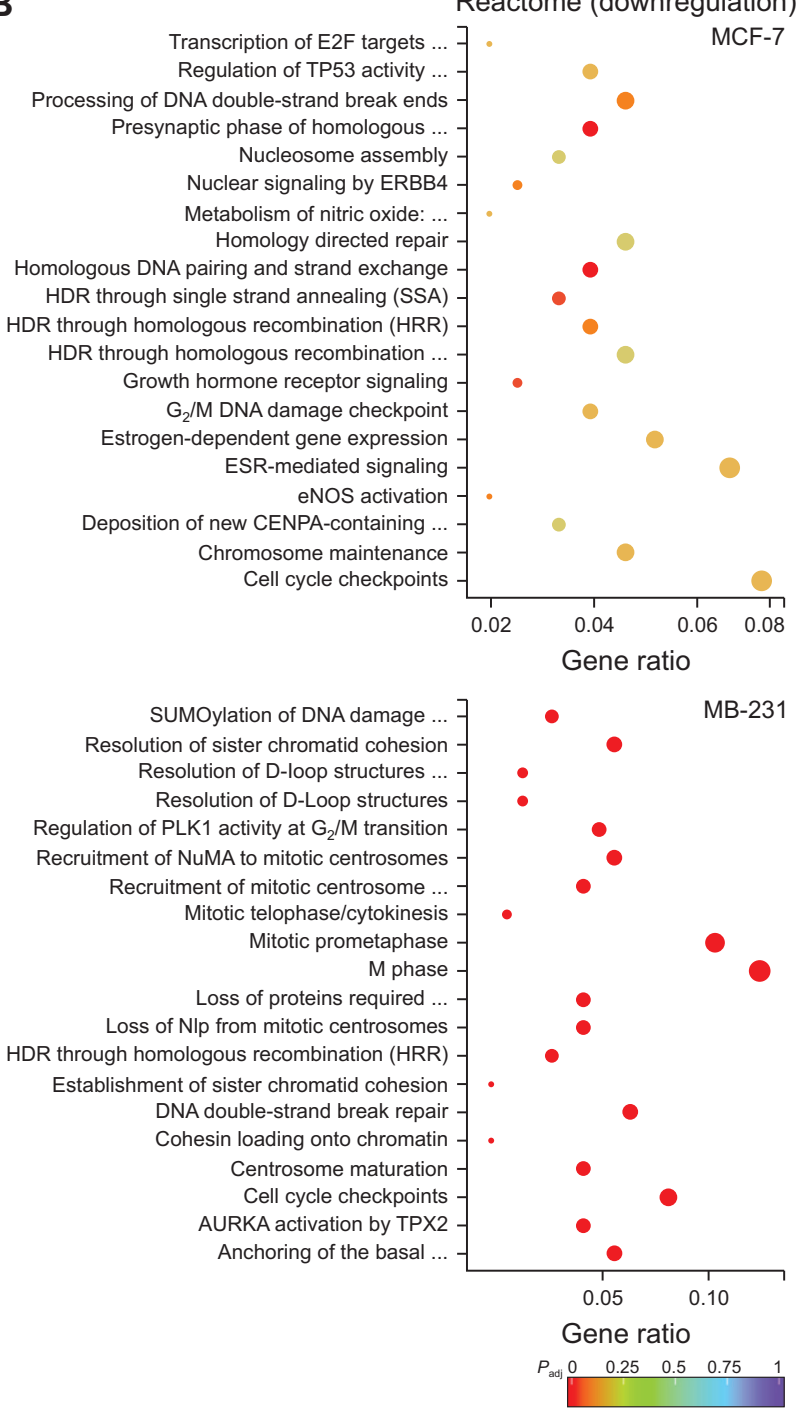

Figure 2. Reactome enrichment analysis for differentially expressed genes by DATS treatment in human breast cancer cells. (A, B) Reactome enrichment analysis of (A) upregulated or (B) downregulated genes by DATS exposure in MCF-7 and MDA-MB-231 cells. DATS, diallyl trisulfide; MCF-7, michigan cancer foundation-7; MDA-MB-231, MD anderson-metastatic breast-231; TNFR, tumor necrosis factor receptor; SRP, signal-recognition particle; CENPA, centromere protein A; AURKA, aurora kinase A; TPX2, targeting protein for Xklp2; $P_{\text {adj; }}$, adjusted $P$-value. 
Kyoto Encyclopedia of Genes and Genomes (KEGG) pathway analyses. The RNA-seq data presented in this study have been submitted to the Gene Expression Omnibus of the National Center for Biotechnology Information and can be retrieved by accession number GSE173616.

\section{Western blotting}

The MCF-7 or MDA-MB-231 cells were plated in 6-cm dishes at a density of $5 \times 10^{5}$ cells/dish and allowed to attach. The cells were treated with DMSO (control) or DATS (10 or 20 $\mu \mathrm{mol} / \mathrm{L}$ ) for 4,8 , and 16 hours. The whole-cell lysates were prepared as described by us previously [18].

A
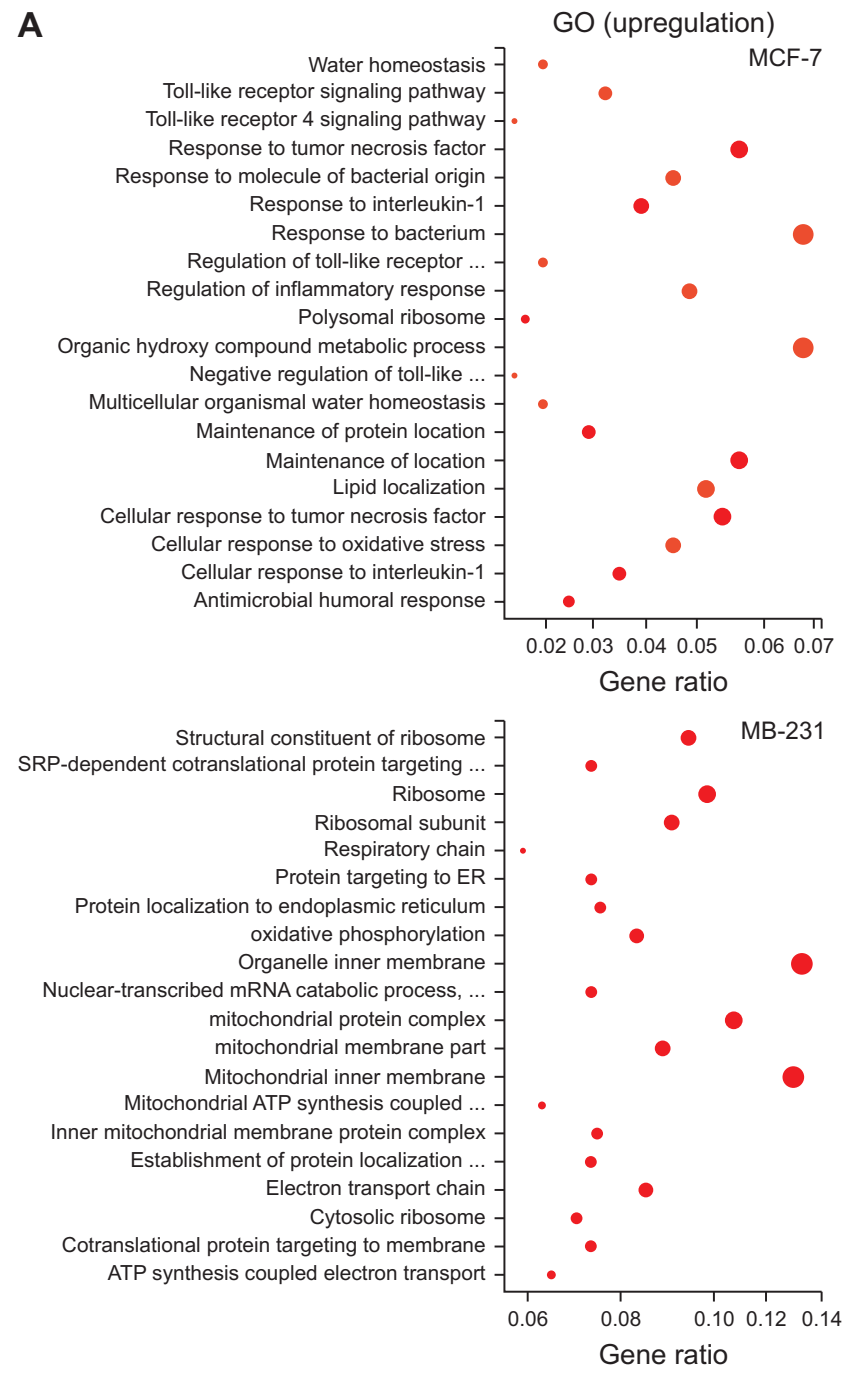

\section{Cell cycle analysis}

The cells $\left(5 \times 10^{5}\right.$ cells/dish) were plated onto 6 -cm dishes in triplicate and incubated overnight and then treated with DMSO (control) or DATS (10 or $20 \mu \mathrm{mol} / \mathrm{L}$ ) for 4 and 8 hours. The cells were then collected by trypsinization and fixed with $70 \%$ ethanol for overnight at $4^{\circ} \mathrm{C}$. Following permeabilization with Triton X-100, the cells were incubated with Alexa Fluor 488-conjugated phospho-(Ser10) histone $\mathrm{H} 3$ antibody and then stained with PI. The cells were analyzed using Accuri C6 flow cytometer.

\section{Statistical analysis}

GraphPad Prism (version 8.0.0) was used to perform statistical analyses. One-way ANOVA followed by Dunnett's test

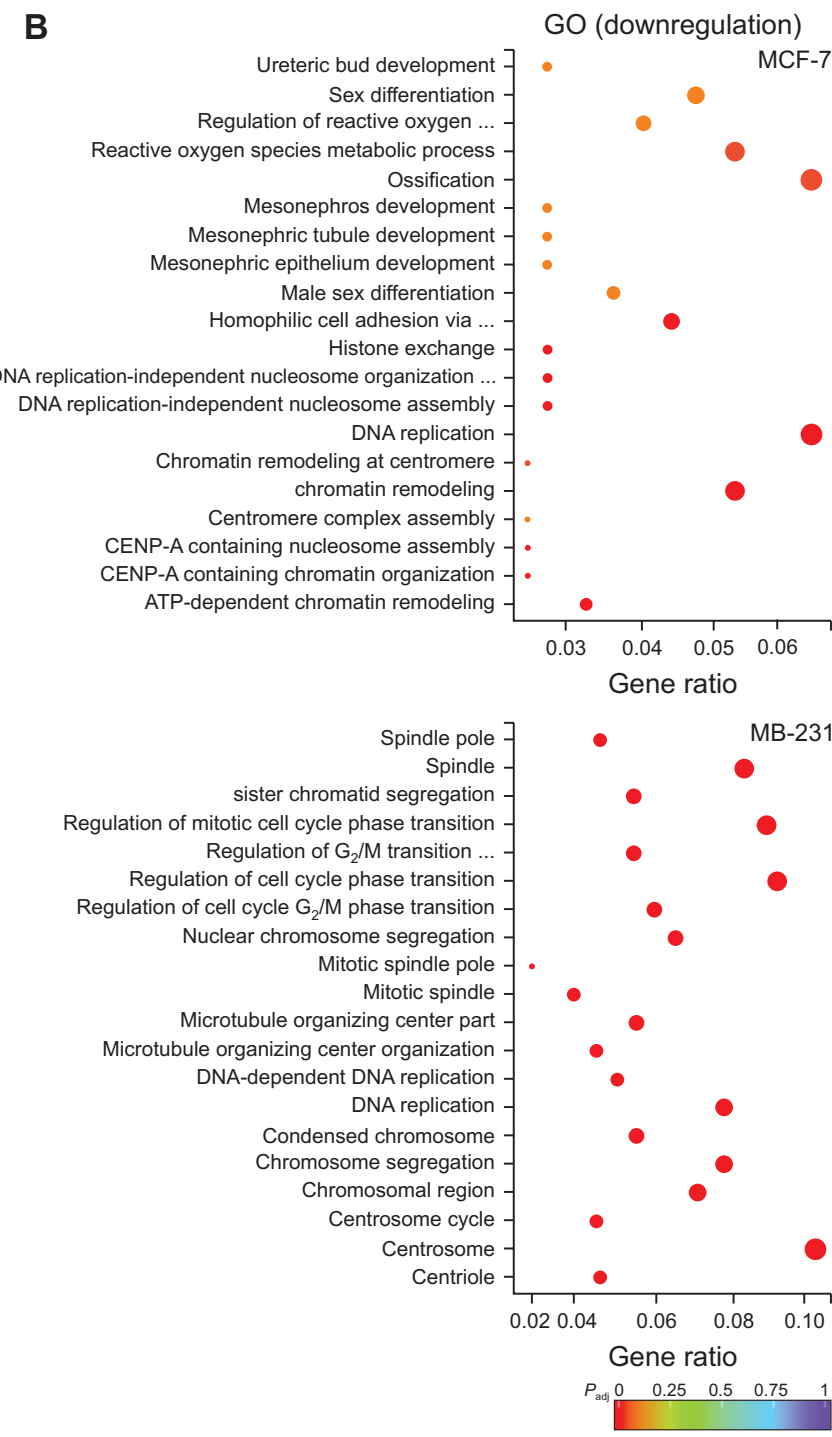

Figure 3. Gene Ontology (GO) enrichment analysis for differentially expressed genes by DATS treatment in human breast cancer cells. (A, B) GO enrichment analysis of $(A)$ upregulated or $(B)$ downregulated genes by DATS exposure in MCF-7 and MDA-MB-231 cells. DATS, diallyl trisulfide; MCF-7, michigan cancer foundation-7; MDA-MB-231, MD anderson-metastatic breast-231; ER, estrogen receptor; CENP-A, centromere protein A; $P_{\text {adj }}$, adjusted $P$-value. 
was used for dose-response analysis. A $P$-value of $<0.05$ was considered statistically significant.

\section{RESULTS}

\section{RNA-seq analysis}

We have shown previously that the viability of MCF-7 and MDA-MB-231 cells is inhibited significantly following 24-hour treatment with 20 and $40 \mu \mathrm{mol} / \mathrm{L}$ DATS (its chemical structure shown in Fig. 1A) [19]. The pharmacokinetic parameters for DATS have been determined in rats after intravenous administration of a single $10 \mathrm{mg}$ dose [20]. The maximum blood concentration of DATS was found to be $\sim 31 \mu \mathrm{mol} / \mathrm{L}$ [20]. Therefore, MCF-7 and MDA-MB-231 cells were treated with
$20 \mu \mathrm{mol} / \mathrm{L}$ DATS for 16 hours prior to RNA isolation and RNAseq analysis. The total mapping rates for the control and DATS-treated MCF-7 cells were about $96.12 \% \pm 0.16 \%$ and $95.93 \% \pm 0.12 \%$, respectively. The total mapping rates for the control and DATS-treated MDA-MB-231 cells were about $96.61 \% \pm 0.21 \%$ and $96.46 \% \pm 0.12 \%$, respectively.

Figure 1B shows Volcano plots that visualize the distribution of differentially expressed genes between control and DATS-treated cells with an adjusted $P$-value of $<0.05$. DATS treatment resulted in upregulation of 539 and 644 genes in MCF-7 and MDA-MB-231 cells, respectively, when compared to corresponding control cells (Fig. 1B). Downregulation of 322 and 508 genes was observed following DATS treatment in MCF-7 and MDA-MB-231 cells, respectively, in comparison
A
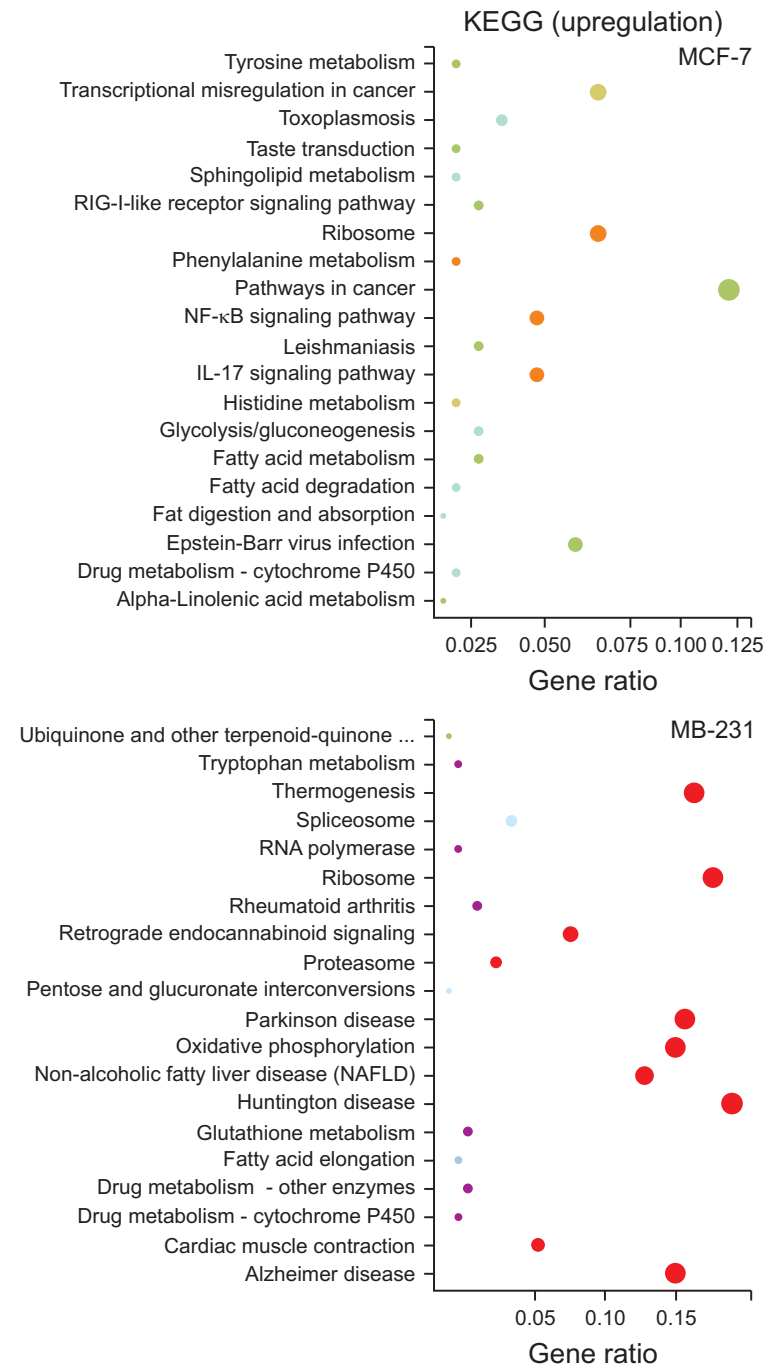

B
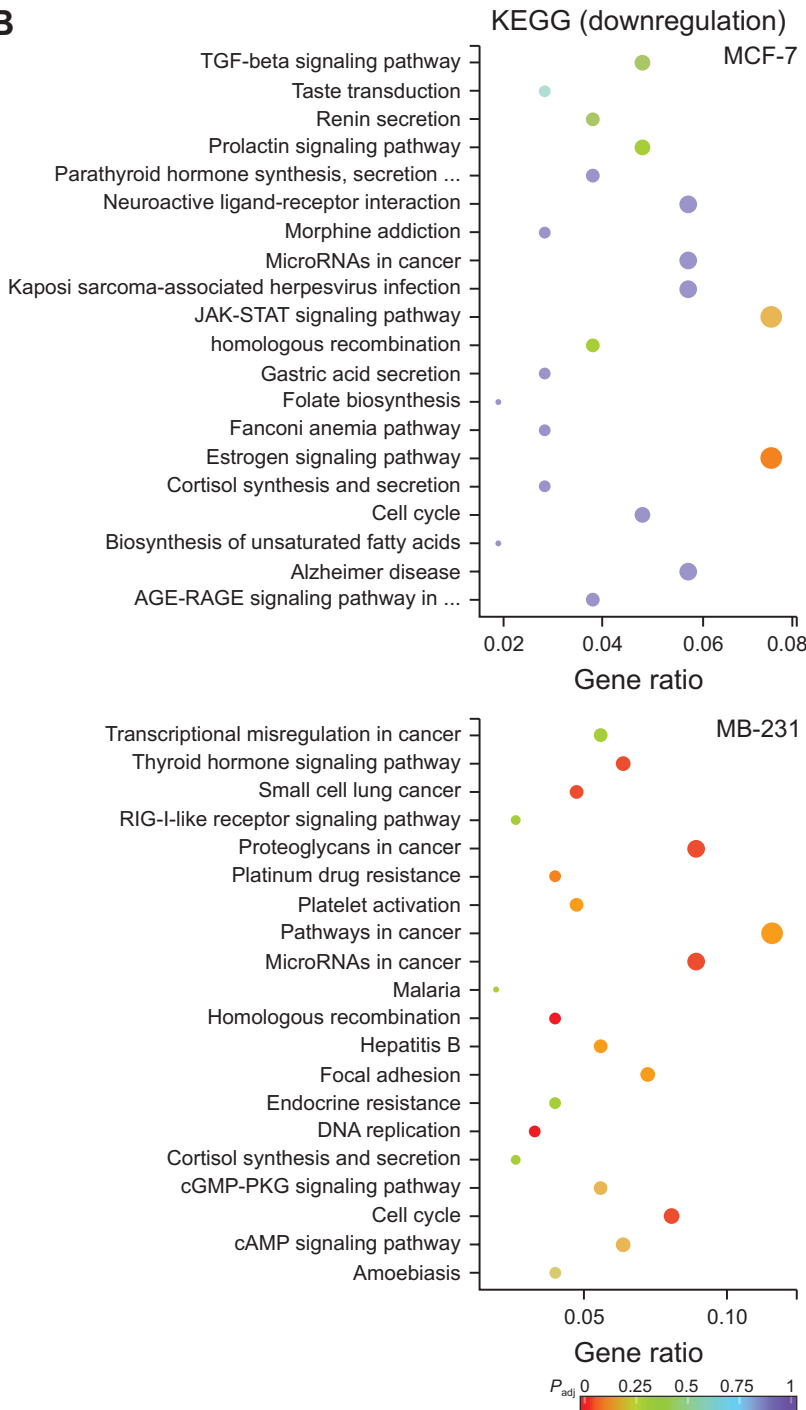

Figure 4. Kyoto Encyclopedia of Genes and Genomes (KEGG) pathway enrichment analysis for differentially expressed genes by DATS treatment in human breast cancer cells. (A, B) KEGG pathway enrichment analysis of (A) upregulated or (B) downregulated genes by DATS exposure in MCF-7 and MDA-MB-231 cells. DATS, diallyl trisulfide; MCF-7, michigan cancer foundation-7; MDA-MB-231, MD anderson-metastatic breast-231; $P_{\text {adj }}$, adjusted $P$-value. 
with corresponding control cells (Fig. 1B). The Venn diagram revealed unique and overlapping gene expression between control and DATS-treatment groups (Fig. 1C). The heatmaps of three replicates of control and DATS-treated samples exhibited highly consistent transcriptional changes (Fig. 1D).

\section{Pathway analyses}

Figure 2 shows the results of the Reactome pathway analysis of the RNA-seq data. The Reactome database covers annotations for a diverse set of molecular and cell biological topics, including cell cycle, metabolism, signaling, transport, cell motility, immune function, host-virus interaction, and neural function. In MCF-7 cells, the top 5 pathways with DATS-mediated upregulation of genes included signaling by interleukins, neutrophil degranulation, regulation of expression of SLITs and ROBOs, interleukin-4 and interleukin-13 signaling, and influenza virus transcription and replication (Fig. 2A). The top 5 pathways with upregulation of genes following DATS treatment in MDA-MB-231 cells were signaling by ROBO receptors, regulation of expression of SLITs and ROBOs, influenza infection, influenza life cycle, and influenza viral transcription and replication (Fig. 2A). In MCF-7 cells, the top 5 pathways with DATS-mediated downregulation of genes included cell cycle checkpoints, ESR-mediated signaling, estrogen-dependent gene expression, chromosome maintenance, and processing of DNA double-strand break (Fig. 2B). The top 5 pathways with downregulation of genes following DATS treatment in MDA-MB-231 cells were M phase, mitotic prometaphase, cell cycle checkpoints, DNA double-strand break repair, and resolution of sister chromatid cohesion (Fig. 2B). The results of GO enrichment analysis, which all three ontologies (cellular component, molecular function, and biological processes), are shown in Figure 3. The KEGG pathway analysis identifies changes in expression of genes associated with metabolic pathway, oxidative phosphorylation, cell cycle, ubiquitin-mediated proteolysis, and so forth. The results of the KEGG pathway analysis are shown in Figure 4 signifying changes in gene expression following DATS treatment in MCF-7 and MDA-MB-231 cells.

\section{The effect of DATS treatment on protein levels of SLIT2 ligand and ROBO1 receptor}

The SLIT/ROBO signaling functions to suppress tumor in breast and other solid tumors [21,22]. Because upregulation of genes associated with the SLIT/ROBO pathway was observed following DATS treatment in both MCF-7 and MDAMB-231 cells in the Reactome pathway analysis, we focused on this pathway for validation of the RNA-seq data. Expression of SLIT2 or ROBO1 protein was not altered meaningfully in either cell line (Fig. 5). These results ruled out a role for the SLIT/ROBO pathway in antitumor effects of DATS.

\section{The effect of DATS treatment on cell cycle progression}

The flow histograms for cell cycle distribution in control and DATS-treated MCF-7 and MDA-MB-231 cells are shown in Figure $6 A$. The fraction of $G_{2} / M$ phase cells was increased significantly after DATS treatment in both cell lines (Fig. 6B). Flow histograms for levels of Ser10 phosphorylated histone $\mathrm{H} 3$, a marker of cells in mitosis phase, are shown in Figure $6 C$. DATS treatment also caused an increase in Ser10 phosphorylation of histone $\mathrm{H} 3$ in both cell lines when compared to control cells (Fig. 6D). These results confirmed $G_{2}$ and $M$ phase cell cycle arrest upon DATS treatment in MCF-7 and MDA-MB-231 cells.

\section{DISCUSSION}

In the present study, we used human breast cancer cell lines representative of two major subtypes to determine DATS-mediated changes in gene expression. Several cancer-relevant pathways are affected by DATS treatment in both cell lines

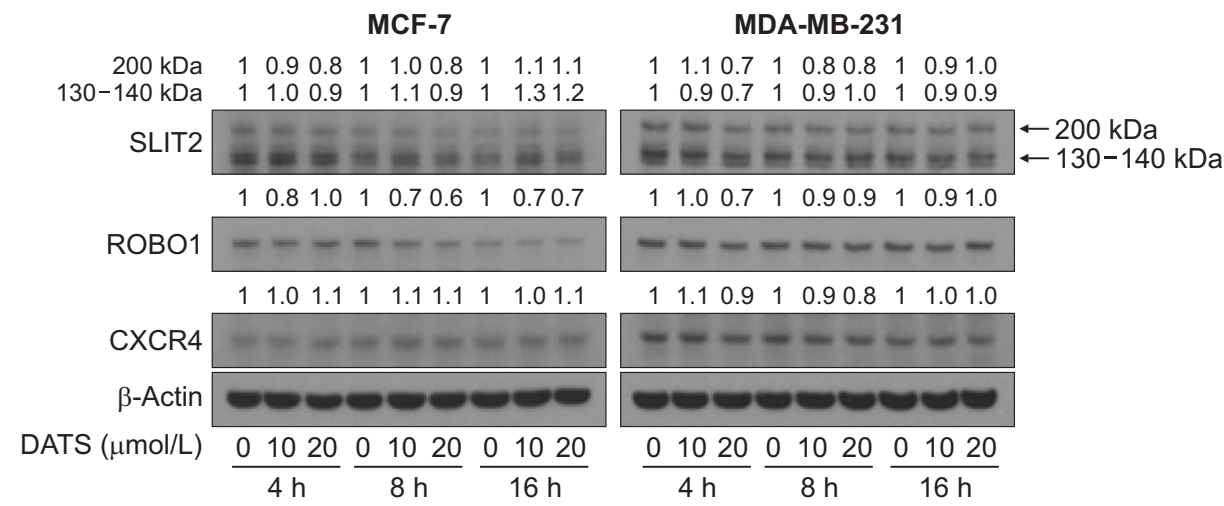

Figure 5. Effect of DATS treatment on the expression of SLIT2/ROBO1 signaling-related proteins. Immunoblot analysis for SLIT2, ROBO1, CXCR4, and $\beta$-Actin proteins using lysates from MCF-7 and MDA-MB-231 cells treated with either DMSO (control) or desired doses of DATS for indicated time points. Numbers above bands are fold change of each protein relative to corresponding DMSO-treated control. Experiments were repeated twice with comparable results. Molecular weights for the observed bands for SLIT2 were indicated. DATS, diallyl trisulfide; MCF-7, michigan cancer foundation-7; MDA-MB-231, MD anderson-metastatic breast-231; CXCR4, C-X-C motif chemokine receptor 4; DMSO, dimethyl sulfoxide. 

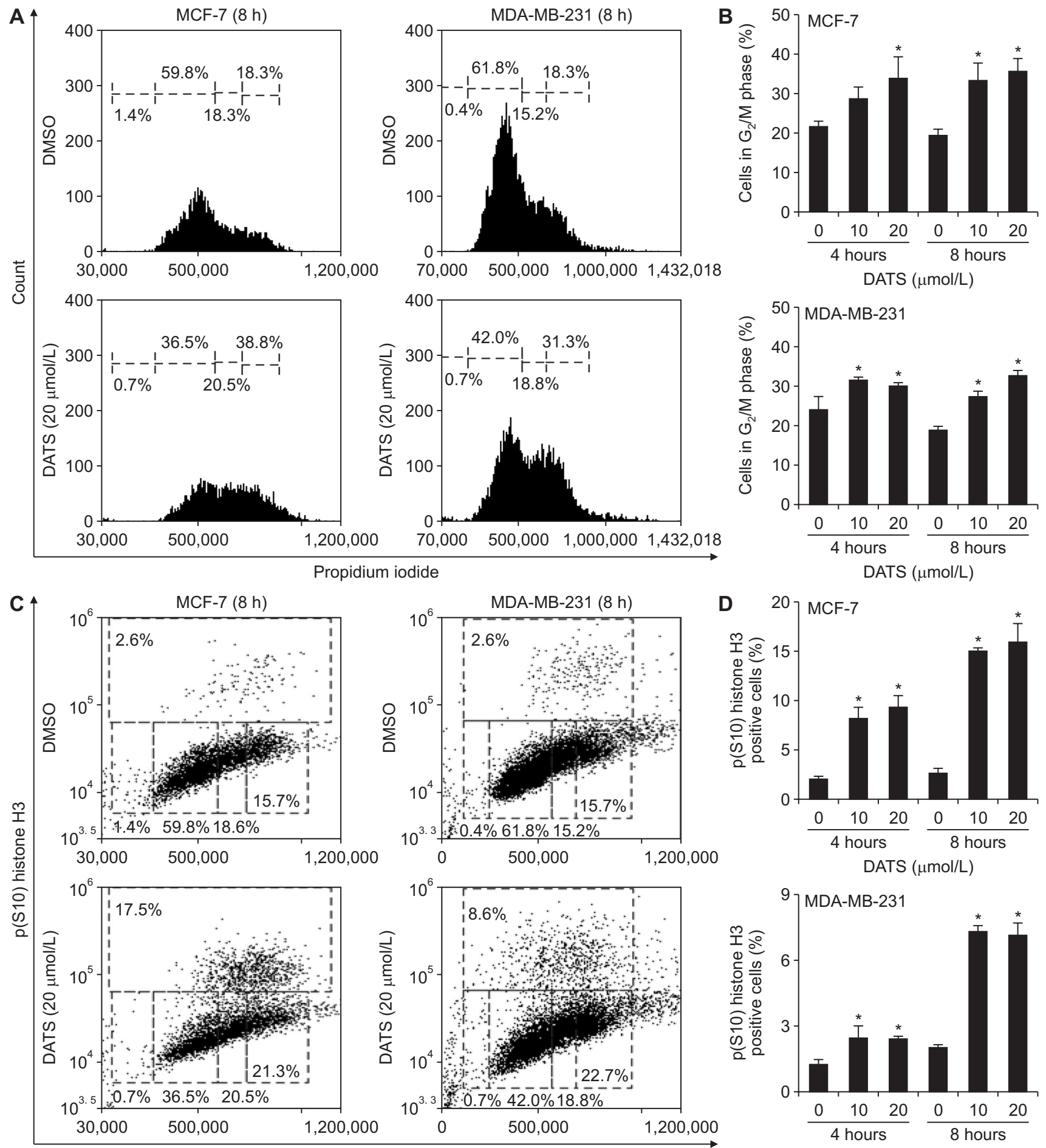

Propidium iodide

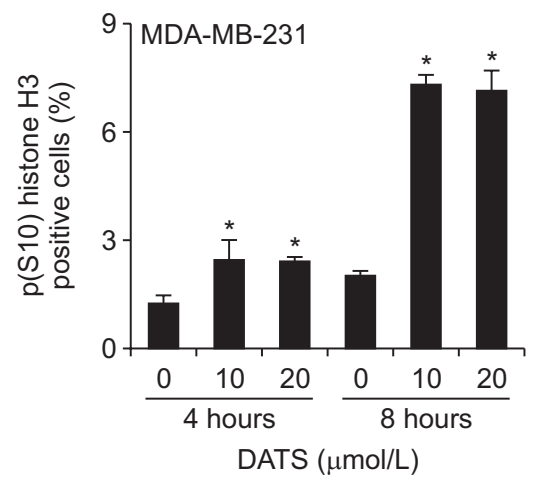

Figure 6. DATS arrested cells at $\mathrm{G}_{2}$ as well as mitotic phase in human breast cancer cells. (A) Representative propidium iodide (PI) histogram displaying $2 \mathrm{~N}$ and $4 \mathrm{~N}$ DNA content in MCF-7 and MDA-MB-231 cells after 8-hour treatment with DMSO (control) or $20 \mu \mathrm{mol} / \mathrm{L}$ DATS. (B) Quantification of the percentage of cells at $\mathrm{G}_{2}$ and M phases in MCF-7 and MDA-MB-231 cells treated with DMSO (control) or indicated doses of DATS for 4 or 8 hours. Results shown are mean \pm SD $(n=3)$ and statistical analysis was done by one-way ANOVA followed by Dunnett's test ${ }^{*} P$ $<0.05$ ). (C) Representative cell cycle histogram of DNA stained with PI and mitotic marker in MCF-7 and MDA-MB-231 cells after 8-hour treatment with DMSO (control) or $20 \mu \mathrm{mol} / \mathrm{L}$ DATS. (D) Quantification of the percentage of cells undergoing mitosis in MCF-7 and MDA-MB-231 cells treated with DMSO (control) or indicated doses of DATS for 4 or 8 hours. Results shown are mean \pm SD $(n=3)$ and statistical analysis was done by one-way ANOVA followed by Dunnett's test $\left({ }^{*} P<0.05\right)$. DATS, diallyl trisulfide; MCF-7, michigan cancer foundation-7; MDA-MB-231, MD anderson-metastatic breast-231; DMSO, dimethyl sulfoxide. 
as revealed by the Reactome, GO, and KEGG pathway analyses. While gene expression changes associated with the SLIT/ROBO pathway did not translate to upregulation of their protein levels after DATS treatment, the $G_{2} / M$ phase cell cycle arrest was consistent with the RNA-seq analysis. We propose that DATS-mediated cell cycle arrest likely contributes to the antitumor effect of DATS. However, it remains to be seen whether DATS-mediated cell cycle arrest is selective for cancer cells and does not occur in normal mammary epithelial cells. It is equally important to determine if DATS treatment causes $\mathrm{G}_{2} / \mathrm{M}$ phase cell cycle arrest in Her2-enriched breast cancer cells. We plan to explore these possibilities in future studies.

The Reactome pathway analysis revealed downregulation of genes associated with ESR (estrogen receptor, ER)-mediated signaling and estrogen-dependent gene expression after DATS treatment in the MCF-7 cell line. The ER- $\alpha$ is epigenetically silenced in the MDA-MB-231 cell line. The RNA-seq data in MCF-7 cells confirm our earlier finding on DATS-mediated suppression of ER- $\alpha$ expression and activity in breast cancer cells [23]. DATS is a relatively more potent suppressor of ER- $\alpha$ protein expression than DAS or DADS [23]. The DATS treatment also suppresses $17 \beta$-estradiol (E2)-induced expression of pS2 and cyclin D1 that are ER- $\alpha$ target gene products [23]. In conclusion, this study identifies ER- $\alpha$ as a novel target of DATS in mammary cancer cells [23].

The present study reveals downregulation of the genes associated with other cancer-relevant signaling pathways potentially contributing to the antitumor effect of DATS. As an example, DATS treatment caused a decrease in expression of genes associated with JAK-STAT signaling pathway (KEGG pathway analysis). The JAK-STAT3 pathway plays an important oncogenic role in breast cancer [24]. We have shown previously that DATS treatment inhibits leptin-stimulated phosphorylation (activation) of STAT3 in MCF-7 and MDA-MB-231 cells [25]. More importantly, Ser727 phosphorylation was decreased significantly by DATS administration in vivo in a triple-negative xenograft model of basal-like SUM159 cells [25].

We have shown previously that DATS inhibits self-renewal of breast cancer stem-like cells (bCSC) in MCF-7 and SUM159 cells [26]. Inhibition of bCSC by DATS was accompanied by downregulation of Forkhead box Q1 (FoxQ1) protein [26]. Overexpression of FoxQ1 in both MCF-7 and SUM159 cell lines conferred significant protection against DATS-mediated inhibition of bCSC population [26]. Surprisingly, the RNA-seq analysis did not reveal downregulation of FoxQ1 in either MCF-7 or MDA-MB-231 cells (present study, data not shown). However, there were differences in experimental conditions that could explain this discrepancy.

For bCSC work, the cells were treated for 24 hours or 72 hours with 2.5 and/or $5 \mu \mathrm{mol} / \mathrm{L}$ DATS [26]. In the present study, cells were treated for 16 hours with $20 \mu \mathrm{mol} / \mathrm{L}$ DATS. Nevertheless, among several stemness-related genes, the expression of pre-B-cell leukemia transcription factor 1 (PBX1) was significantly downregulated by DATS treatment in both MCF-7 and MDA-MB-231 cells (data not shown). Overexpression of $\mathrm{PBX} 1$ has been reported in the stroma of invasive breast cancer, and ductal and invasive lobular breast cancers when compared to normal mammary tissue [27]. Moreover, a high PBX1 expression was associated with poor prognosis in ER+ breast cancer patients [27]. Additional significantly $(P=0.05$ or lower) downregulated genes in DATS-treated MDA-MB-231 cells included ARID1A, CNOT1, HIF1A, JAG1, LEO1, SETD2, SEMA3C, and ZC3H13 (data not shown). Further work is necessary to determine the significance of these genes in bCSC inhibition by DATS.

In conclusion, the present study identifies signaling pathways that may contribute to the antitumor effect of DATS. However, additional work is needed to validate other gene expression changes following DATS treatment based on the results of the Reactome, GO, and KEGG pathway analyses.

\section{ACKNOWLEDGMENTS}

This study was supported by the National Cancer Institute at the National Institutes of Health grant R01 CA219180 (to SVS). This study used the UPMC Hillman Cancer Center Flow Cytometry Facility supported by the National Cancer Institute at the National Institutes of Health grant P30 CA047904.

\section{CONFLICTS OF INTEREST}

No potential conflicts of interest were disclosed.

\section{ORCID}

Eun-Ryeong Hahm, https://orcid.org/0000-0002-3197-4575 Su-Hyeong Kim, https://orcid.org/0000-0003-2071-613X Sivapar V. Mathan, https://orcid.org/0000-0002-3642-656X Rana P. Singh, https://orcid.org/0000-0003-4261-7044 Shivendra V. Singh, https://orcid.org/0000-0002-3733-144X

\section{REFERENCES}

1. Siegel RL, Miller KD, Fuchs HE, Jemal A. Cancer statistics, 2021. CA Cancer J Clin 2021;71:7-33.

2. Farkas A, Vanderberg R, Merriam S, DiNardo D. Breast cancer chemoprevention: a practical guide for the primary care provider. J Womens Health (Larchmt) 2020;29:46-56.

3. Sørlie T, Perou CM, Tibshirani R, Aas T, Geisler S, Johnsen H, et al. Gene expression patterns of breast carcinomas distinguish tumor subclasses with clinical implications. Proc Natl Acad Sci USA 2001;98:10869-74.

4. Fisher B, Costantino JP, Wickerham DL, Redmond CK, Kavanah M, Cronin WM, et al. Tamoxifen for prevention of breast cancer: report of the National Surgical Adjuvant Breast and Bowel Project 
Hahm et al.

P-1 Study. J Natl Cancer Inst 1998;90:1371-88.

5. Goss PE, Ingle JN, Alés-Martínez JE, Cheung AM, Chlebowski RT, Wactawski-Wende J, et al. Exemestane for breastcancer prevention in postmenopausal women. N Engl J Med 2011;364:2381-91.

6. Kapinova A, Kubatka P, Golubnitschaja O, Kello M, Zubor $\mathrm{P}$, Solar $\mathrm{P}$, et al. Dietary phytochemicals in breast cancer research: anticancer effects and potential utility for effective chemoprevention. Environ Health Prev Med 2018;23:36.

7. Powolny AA, Singh SV. Multitargeted prevention and therapy of cancer by diallyl trisulfide and related Allium vegetable-derived organosulfur compounds. Cancer Lett 2008;269:305-14.

8. Singh SV, Singh K. Cancer chemoprevention with dietary isothiocyanates mature for clinical translational research. Carcinogenesis 2012;33:1833-42.

9. Challier B, Perarnau JM, Viel JF. Garlic, onion and cereal fibre as protective factors for breast cancer: a French case-control study. Eur J Epidemiol 1998;14:737-47.

10. Desai G, Schelske-Santos M, Nazario CM, Rosario-Rosado RV, Mansilla-Rivera I, Ramírez-Marrero F, et al. Onion and garlic intake and breast cancer, a case-control study in Puerto Rico. Nutr Cancer 2020;72:791-800.

11. Pourzand A, Tajaddini A, Pirouzpanah S, Asghari-Jafarabadi M, Samadi N, Ostadrahimi AR, et al. Associations between dietary Allium vegetables and risk of breast cancer: a hospital-based matched case-control study. J Breast Cancer 2016;19:292-300.

12. Dorant E, van den Brandt PA, Goldbohm RA. Allium vegetable consumption, garlic supplement intake, and female breast carcinoma incidence. Breast Cancer Res Treat 1995;33:163-70.

13. Block E. The chemistry of garlic and onions. Sci Am 1985;252:114-9.

14. Xiao D, Choi S, Johnson DE, Vogel VG, Johnson CS, Trump $\mathrm{DL}$, et al. Diallyl trisulfide-induced apoptosis in human prostate cancer cells involves c-Jun N-terminal kinase and extracellularsignal regulated kinase-mediated phosphorylation of $\mathrm{Bcl}-2$. Oncogene 2004;23:5594-606.

15. Na HK, Kim EH, Choi MA, Park JM, Kim DH, Surh YJ. Diallyl trisulfide induces apoptosis in human breast cancer cells through ROS-mediated activation of JNK and AP-1. Biochem Pharmacol 2012;84:1241-50.
16. Yu TH, Wu CM, Liou YC. Volatile compounds from garlic. J Agric Food Chem 1989;37:725-30.

17. Kim SH, Hahm ER, Singh KB, Shiva S, Stewart-Ornstein J, Singh SV. RNA-seq reveals novel mechanistic targets of withaferin $A$ in prostate cancer cells. Carcinogenesis 2020;41:778-89.

18. Xiao D, Srivastava SK, Lew KL, Zeng Y, Hershberger P, Johnson $\mathrm{CS}$, et al. Allyl isothiocyanate, a constituent of cruciferous vegetables, inhibits proliferation of human prostate cancer cells by causing G2/M arrest and inducing apoptosis. Carcinogenesis 2003;24:891-7.

19. Chandra-Kuntal K, Lee J, Singh SV. Critical role for reactive oxygen species in apoptosis induction and cell migration inhibition by diallyl trisulfide, a cancer chemopreventive component of garlic. Breast Cancer Res Treat 2013;138:69-79.

20. Sun X, Guo T, He J, Zhao M, Yan M, Cui F, et al. Determination of the concentration of diallyl trisulfide in rat whole blood using gas chromatography with electron-capture detection and identification of its major metabolite with gas chromatography mass spectrometry. Yakugaku Zasshi 2006;126:521-7.

21. Gu F, Ma Y, Zhang J, Qin F, Fu L. Function of Slit/Robo signaling in breast cancer. Front Med 2015;9:431-6.

22. Jiang Z, Liang G, Xiao Y, Qin T, Chen X, Wu E, et al. Targeting the SLIT/ROBO pathway in tumor progression: molecular mechanisms and therapeutic perspectives. Ther Adv Med Oncol 2019;11:1758835919855238.

23. Hahm ER, Singh SV. Diallyl trisulfide inhibits estrogen receptor- $\alpha$ activity in human breast cancer cells. Breast Cancer Res Treat 2014;144:47-57.

24. Banerjee $\mathrm{K}$, Resat $\mathrm{H}$. Constitutive activation of STAT3 in breast cancer cells: a review. Int J Cancer 2016;138:2570-8.

25. Kim SH, Hahm ER, Singh KB, Singh SV. Diallyl trisulfide inhibits leptin-induced oncogenic signaling in human breast cancer cells but fails to prevent chemically-induced luminal-type cancer in rats. J Cancer Prev 2020;25:1-12.

26. Kim SH, Kaschula CH, Priedigkeit N, Lee AV, Singh SV. Forkhead box $\mathrm{Q} 1$ is a novel target of breast cancer stem cell inhibition by diallyl trisulfide. J Biol Chem 2016;291:13495-508.

27. Ao X, Ding W, Ge H, Zhang Y, Ding D, Liu Y. PBX1 is a valuable prognostic biomarker for patients with breast cancer. Exp Ther Med 2020;20:385-94. 